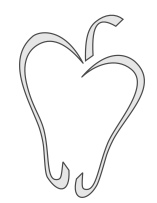

ISSN 1852-4249

\title{
Self-sustained oscillations with delayed velocity feedback
}

\author{
D. H. Zanette ${ }^{1 *}$
}

\begin{abstract}
We study a model for a nonlinear mechanical oscillator, relevant to the dynamics of microand nanomechanical time-keeping devices, where periodic motion is sustained by a feedback force proportional to the oscillation velocity. Specifically, we focus our attention on the effect of a time delay in the feedback loop, assumed to originate in the electric circuit that creates and injects the self-sustaining force. Stationary oscillating solutions to the equation of motion, whose stability is insured by the crucial role of nonlinearity, are analytically obtained through suitable approximations. We show that a delay within the order of the oscillation period can suppress self-sustained oscillations. Numerical solutions are used to validate the analytical approximations.
\end{abstract}

\section{Introduction}

Inside any modern time-keeping device, the principal component is an oscillator which autonomously generates a stationary periodic signal with a welldefined frequency. The only external input to the system is the power needed to sustain the oscillations. In devices based on mechanical oscillators -which comprise essentially all present-day clocks, with the exception of the atomic kind--, sustained periodic motion is achieved by forcing the oscillator with a conditioned version of the signal generated by the oscillator itself [1]. If this reinjected force is in-phase with the oscillation velocity, the resonant response is maximal, thus optimizing power consumption during the process of signal conditioning.

Purely mechanical devices employed this feedback scheme already in the Middle Ages. In fact, the escapement mechanism was routinely used in

*E-mail: zanette@cab.cnea.gov.ar

1 Centro Atómico Bariloche and Instituto Balseiro (Comisión Nacional de Energía Atómica, Universidad Nacional de Cuyo), Consejo Nacional de Investigaciones Científicas y Técnicas, Avda. E. Bustillo 9500, 8400 San Carlos de Bariloche, Río Negro, Argentina. clock building since the 13 th century. In modern clocks, oscillators are built from synthetic quartz crystals and feedback is implemented electronically. At the micro- and nanoscale, quartz crystals are expected to be replaced by simpler mechanical oscillators such as tiny vibrating silica beams $[2,3]$, which are easily built during circuit printing and can be actuated by very small electric fields $[4,5]$.

In a series of recent experiments on micromechanical oscillators, it has been shown that selfsustained oscillations can be achieved with a feedback force proportional to the oscillation velocity [6]. Since a purely linear mechanical system cannot display stable periodic motion, this kind of feedback force must necessarily be compensated by some nonlinear contribution from the oscillator dynamics itself. In the experiments, this balance turned out to come from the damping force, which was proportional to the velocity but showed a nonlinear dependence on the oscillation amplitude.

In the present paper, we analyze a model for selfsustained periodic motion in a mechanical oscillator subjected to a feedback force proportional to the velocity, and nonlinear amplitude-dependent terms both in damping and in the restoring force. Emphasis is put on the effects of time delays in the 
Papers in Physics, vol. 9, ART. 090003 (2017) / D. H. Zanette

feedback circuit, which modify the phase shift between the feedback force and the velocity. It is shown that these delays affect the response of the oscillator, to the point that stable periodic motion can even be suppressed. The model is studied analytically within suitable approximations, and the results are compared with numerical solutions to the equation of motion.

\section{Mechanical model for self- sustained oscillations}

Our model is based on an equation of motion for a one-dimensional variable $x(t)$, which represents the departure from equilibrium of a mechanical oscillator:

$$
\ddot{x}+\mu\left(1+\alpha x^{2}\right) \dot{x}+\left(1+\beta x^{2}\right) x=g \dot{x}(t-\tau),
$$

where $\mu$ is the damping coefficient per unit mass. The coefficients $\alpha$ and $\beta$ weight the amplitudedependent nonlinear corrections to the damping force and to the elastic force, respectively. The former is a Van der Pol-like nonlinearity, while the cubic contribution to the restoring force defines a Duffing oscillator [7]. Both kinds of nonlinearity, with $\alpha, \beta>0$, have been experimentally verified to occur in micromechanical oscillators formed by silica beams clamped at their two ends (clampedclamped, or c-c beams $[4,6])$. In the main oscillation mode, $\mathrm{c}-\mathrm{c}$ beams vibrate much like a plucked string, so that $x(t)$ can be associated with the displacement of the middle point of the beam with respect to its rest position.

The right-hand side of Eq. (1) represents the feedback force per unit mass, which is proportional to the velocity at the delayed time $t-\tau$. As stated in the introduction, this delay is expected to originate in the electric circuit that reads, conditions, and reinjects the oscillator signal, due to the time elapsed during signal processing. Although $\tau$ should be a very short time, it is not necessarily negligible as compared with other time scales in the system, in particular with the oscillation period. In fact, micro- and nano-oscillators vibrate with frequencies from the order of $100 \mathrm{kHz}$ [4] to $1 \mathrm{GHz}$ [8].

Time units in Eq. (1) have been chosen in such a way that the natural frequency of the undamped $(\mu=0)$, linear $(\beta=0)$, unforced $(g=0)$ oscillator equals unity. Meanwhile, the units of $x$ can be fixed in such a way that the coefficient $\alpha$ adopts any prescribed value. Hence, without generality loss and for future convenience, we fix $\alpha=4$.

\section{i. Approximate stationary solutions}

Nonlinearity in Eq. (1) prevents obtaining an exact solution. Approximate stationary solutions can be found by the standard procedure of neglecting higher-harmonic contributions to the oscillations [7]. Within this approximation, and proposing $x(t)=\frac{1}{2} A \exp (i \omega t)+$ c.c., we get a complex algebraic equation whose real and imaginary parts read

$$
1-\omega^{2}+b A^{2}=g \omega \sin \omega \tau,
$$

with $b=3 \beta / 4$, and

$$
\mu\left(1+A^{2}\right)=g \cos \omega \tau
$$

These are equations for the unknowns $A$ and $\omega$, which have been obtained assuming $A, \omega \neq 0$.

Note that, to have a non-negative solution for $A^{2}$, Eq. (3) requires that $g \geq \mu$. Physically, this amounts to require that the energy input from the feedback force is not less than the energy dissipated by damping. Otherwise, stationary periodic motion cannot be sustained. In the following, thus, we work under the assumption that such condition holds.

Squaring and summing up Eqs. (2) and (3), we obtain

$$
\left(1-\omega^{2}+b A^{2}\right)^{2}+\mu^{2} \omega^{2}\left(1+A^{2}\right)^{2}=g^{2} \omega^{2},
$$

namely, a relation between the oscillation amplitude $A$ and the frequency $\omega$ which does not involve explicitly the delay $\tau$. Black (full and dotted) lines in Fig. 1 represent this relation for the parameters indicated in the figure.

The graph of the relation between amplitude and frequency expressed by Eq. (4) can be interpreted as a resonance curve, in the sense that it characterizes the response of the oscillator to the feedback force. In fact, its shape resembles the upper part 


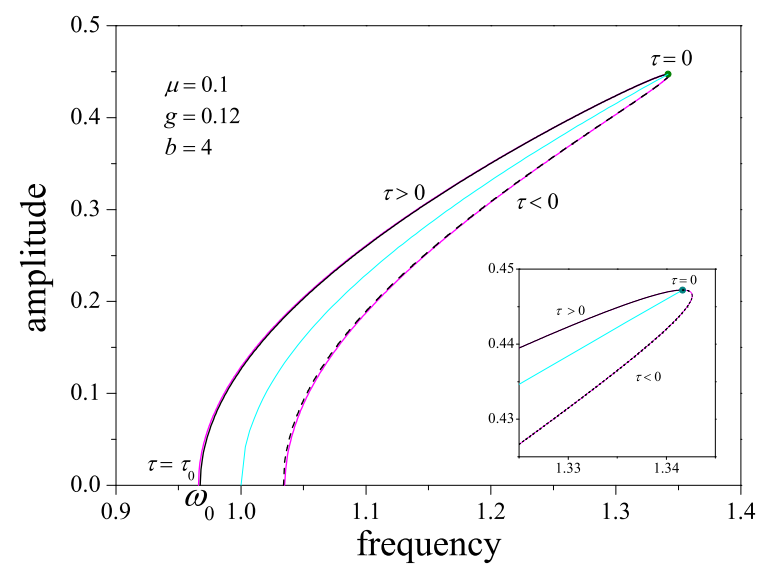

Figure 1: Amplitude-frequency relation, Eq. (4), for $\mu=0.1, g=0.12$, and $b=4$. Full and dotted black lines show the branches where $\tau$ is positive and negative, respectively. The green dot stands at the point of maximal amplitude, corresponding to delay $\tau=0$. For positive delays, oscillations become suppressed for $\tau=\tau_{0}$, at frequency $\omega_{0}$, where the amplitude vanishes. The magenta line is the short-delay approximation, and the cyan line is the backbone curve. The inset shows a close-up around the peak.

of the Duffing resonance curve [7], and its mathematical origin is similar. From the physical point of view, however, it is important to stress that $\omega$ is not a control parameter -as in the standard case of an externally forced system- but emerges as an autonomous dynamical property of the self-sustained oscillator. The parameter whose variation defines the resonance curve of Fig. 1 is, on the other hand, the time delay $\tau$. In the figure, we have highlighted the point corresponding to $\tau=0$ which, as can be seen from Eq. (3), corresponds to the maximum of the amplitude,

$$
A_{\max }=\sqrt{\frac{g}{\mu}-1} .
$$

Note that, due to the smooth profile of the resonance curve at its peak, the frequency at $\tau=0$, given by $\sqrt{1+b A_{\max }^{2}}$, does not equal the maximum frequency attainable by the oscillator, but lies slightly below. This is better appreciated in the figure inset.
Different line types (full and dotted) in Fig. 1 represent the branches of positive and negative $\tau$. Naturally, in an experiment, only the branch of positive time delays can be observed. However, stationary oscillatory solutions exist -and, as we discuss later, are stable-irrespectively of the sign of $\tau$.

\section{ii. Oscillation suppression}

The most relevant dynamical feature emerging from Eqs. (2) and (3) is that, as $\tau$ varies from zero to both positive and negative values, the oscillation amplitude decreases and, eventually, vanishes for sufficiently long delays. This situation is reached at the points where the resonance curve in Fig. 1 intersects the horizontal axis. Focusing on the branch of positive delays, oscillations are suppressed when their frequency reaches

$$
\omega_{0}=\sqrt{1+\Delta-\sqrt{(1+\Delta)^{2}-1}},
$$

with $\Delta=\left(g^{2}-\mu^{2}\right) / 2$, corresponding to a critical delay

$$
\tau_{0}=\omega_{0}^{-1}\left|\arccos \frac{\mu}{g}\right| .
$$

Oscillation suppression can be understood in qualitative terms as a consequence of the increasing phase shift between the feedback force and the velocity when $\tau$ grows. As the phase shift becomes larger, the "timing" of energy supply by feedback fails to counteract energy dissipation by damping. The energy balance -implicit in Eq. (3)- cannot be further maintained, and periodic motion dies out.

Figure 2 shows the critical delay $\tau_{0}$ at which oscillations are suppressed, as a function of the feedback amplitude $g$, and for various values of the damping $\mu$. For $g$ just above $\mu, \tau_{0}$ grows from zero and -if $\mu$ is small enough- attains a plateau around $\pi / 2$. Note that, since the oscillation frequency is always near unity, in this plateau the phase shift between feedback and velocity is also close to $\pi / 2$. In other words, in this zone the feedback force is in-phase with the displacement $x(t)$. For larger values of $g$, $\tau_{0}$ leaves the plateau and grows further.

We point out that very small values of the damping $\mu$, as considered in Fig. 2, are realistic when 
Papers in Physics, vol. 9, ART. 090003 (2017) / D. H. Zanette

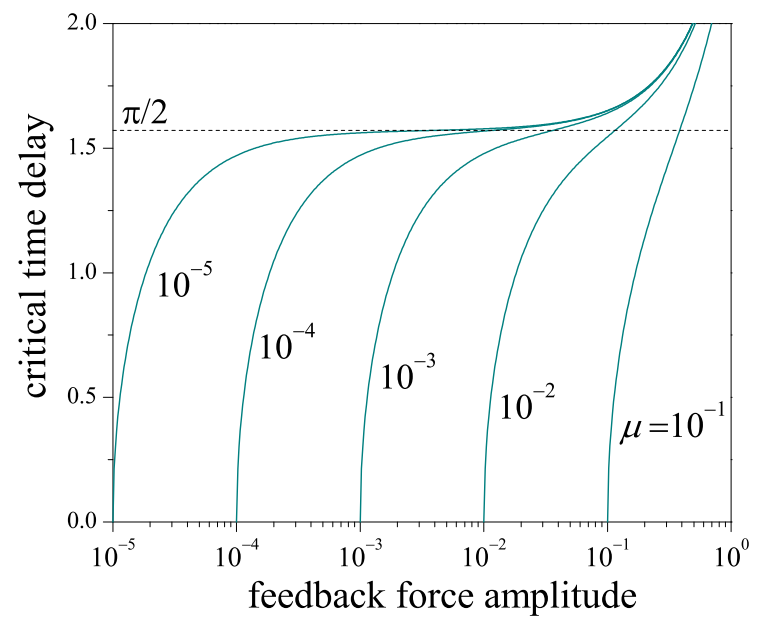

Figure 2: Critical time delay for oscillation suppression, $\tau_{0}$ as given by Eq. (7), vs. the feedback force amplitude $g$, for various values of the damping coefficient $\mu$.

working with micro- and nanomechanical oscillators. Indeed, these devices have quality factors $Q$ $\left(\sim \mu^{-1}\right)$ typically above $10^{4}[4]$. In Fig. 1 , on the other hand, we have chosen a relatively large value of $\mu$ for clarity in the graphical representation. For very small values of $\mu$, in fact, the resonance curve becomes too narrow for its features to be clearly discerned in a plot.

\section{iii. Stability of self-sustained oscillations}

Stability of the stationary oscillatory solutions presented above can be assessed by the method of multiple time scales [7], which provides equations of motion for the relatively slow dynamics of the oscillation amplitude and phase. The method works under the assumption that there is a clear separation between the oscillation period and other time scales involved in the system. In the present case, this condition is fulfilled for small damping, $\mu \ll 1$, as it is energy dissipation which controls the relaxation of amplitude and frequency to their asymptotic values.

Application of the method of multiple scales to Eq. (1) straightforwardly shows that when oscillatory solutions of non-vanishing amplitude do exist, they are stable under arbitrary perturbations to their amplitude and phase (or frequency), irrespec- tively of the sign of the delay. Instead of giving the details of this standard calculation, we resort here to a simple physical argument to explain stability, which sheds useful light on the role of nonlinearity.

Assume that the oscillator is in stationary periodic motion. This implies, in particular, that the energy input from feedback is exactly balanced by dissipation. If motion is now perturbed in such a way that the oscillation amplitude increases, the velocity increases accordingly, thus making feedback and damping to grow in the same proportion (since both are proportional to the velocity). However, due to the amplitude-dependent nonlinear correction in the damping coefficient, there is an extra growth in damping which enhances dissipation and therefore counteracts the perturbation, as the amplitude will tend to decrease. A symmetric argument applies if, on the contrary, the amplitude is perturbed to lower values.

If, on the other hand, the perturbation makes the frequency to increase, the velocity also increases as so do feedback and damping. Through the cubic nonlinearity in the restoring force, however, such change in the frequency leads the amplitude to grow. This growth, in turn, implies an enhancement in dissipation, as explained in the preceding paragraph. Consequently, the perturbation is counteracted by the oscillator response. The case where the frequency decreases is analogous. Under perturbations of both amplitude and frequency, therefore, nonlinearity plays a key role in insuring the stability of stationary oscillations.

\section{Further approximations}

\section{i. Backbone approximation}

As mentioned in section II.ii., a realistic limit when working with micro- and nanomechanical oscillators consists in considering very small values of the damping coefficient $\mu$. In order to insure the existence of physically meaningful solutions for the amplitude and the frequency, however, Eq. (3) requires that the limit of $\mu \rightarrow 0$ is taken along with the limit $g \rightarrow 0$, maintaining a finite ratio $r=g / \mu$. In physical terms, this joint limit is justified by the fact that the smaller the rate of energy dissipation, the smaller the feedback force necessary to maintain oscillatory motion.

In the crudest approximation, the resonance 
Papers in Physics, vol. 9, ART. 090003 (2017) / D. H. Zanette

curve collapses to a single-valued curve with equation

$$
A=\sqrt{\frac{1-\omega^{2}}{b}},
$$

plotted as a cyan line in Fig. 1. This is the so-called backbone approximation to the resonance curve $[7$, 9]. Within this approximation, the time delay as a function of the frequency along the backbone curve is

$$
\tau=\omega^{-1} \arccos \left(\frac{1+b-\omega^{2}}{r b}\right) .
$$

Although, for the relatively large value of $\mu$ considered in Fig. 1, the backbone curve gives a poor approximation to the resonance curve; as $\mu$ becomes smaller the two branches of the latter collapse to the backbone. A measure of this collapse is provided by computing the frequency of oscillation suppression, $\omega_{0}$, in the limit of small damping. Equation (6) yields

$$
\omega_{0} \approx 1-\frac{\mu}{2} \sqrt{r^{2}-1},
$$

showing that the width of the resonance curve at its base $(A=0)$ is proportional to $\mu$. In this limit, the time delay for oscillation suppression is still given by Eq. (7).

\section{ii. Short-delay approximation}

Another relevant approximation, which drastically simplifies the problem of solving Eqs. (2) and (3), is obtained for small values of $\tau$. In fact, approximating $\sin \omega \tau \approx \omega \tau$ and $\cos \omega \tau \approx 1-\omega^{2} \tau^{2} / 2$, we obtain linear equations for $A^{2}$ and $\omega^{2}$, whose solutions yield

$$
A=\sqrt{\frac{2(g-\mu)(1+g \tau)-g \tau^{2}}{2 \mu(1+g \tau)+b g \tau^{2}}}
$$

and

$$
\omega=\sqrt{2 \frac{b(g-\mu)+\mu}{2 \mu(1+g \tau)+b g \tau^{2}}} .
$$

The magenta line in Fig. 1 represents these results. For the parameters of the figure, thus, this is an excellent approximation along the entire resonance curve.

\section{Numerical results}

As a validation of the analytical results obtained by means of the approximation considered in section II., i.e., neglecting the higher-harmonic contributions to oscillatory motion, we have numerically solved Eq. (1) for various parameter sets. We have used a fourth-order Runge-Kutta scheme, with the only non-standard feature that, due to the delay in the feedback force, it is necessary to specify the solution $x(t)$ for all times $-\tau \leq t \leq 0$, instead of the initial condition at $t=0$. In all cases, we have considered a constant value of $x(t)$, hence with $\dot{x}(t)=0$, in that interval.

For all the parameter sets taken into account in the numerical calculations, we have found that, after a transient time which scaled as $\mu^{-1}$, the oscillator reached periodic motion. This observation is in qualitative agreement with our argument of section II.iii., which predicts stability of periodic motion under very general conditions.

A more quantitative comparison between theoretical and numerical results, illustrated for the parameter set of Fig. 1, is given in Fig. 3. The main panel shows, as green dots, numerical measurements of the frequency and amplitude of long-time periodic motion for various (positive) values of the delay in the interval $0 \leq \tau \leq 0.4$. The black full line stands for the theoretical result. The inset shows the same data in the amplitude-delay plane. We see that the agreement is generally very good. Not unexpectedly, the theoretical approximation improves towards smaller amplitudes, where nonlinear effects are weaker and periodic motion is better described by a pure harmonic oscillation, as assumed in section II. As a matter of fact, Fig. 3 is limited to amplitudes above 0.25 since, in the plot, theoretical and numerical results are indistinguishable for smaller values.

The agreement between our theoretical and numerical results rises the question to what extent higher-harmonic components, which are a direct product of nonlinearity but have been neglected in our analytical approximation, constitute a substan- 
Papers in Physics, vol. 9, ART. 090003 (2017) / D. H. Zanette

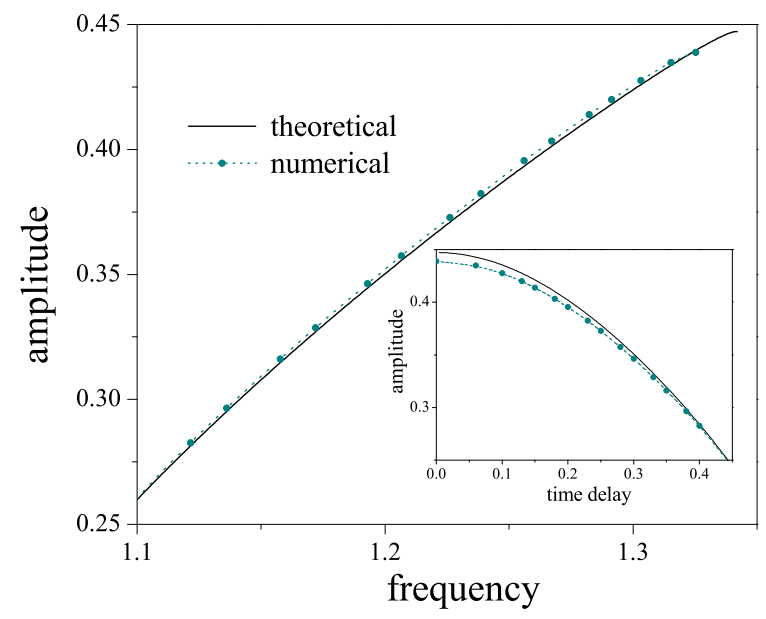

Figure 3: Theoretical (black line) and numerical (green dots) results for the amplitude-frequency relation of periodic oscillatory motion, with $\mu=0.1$, $g=0.12$, and $b=4$, and for various values of the delay in the interval $[0,0.4]$. The inset shows the same data in the amplitude-delay domain. Dotted green lines have been plotted as a guide to the eye.

tial contribution to oscillatory motion. To evaluate this, we calculate the amplitudes of different Fourier components in the long-time numerical solution for $x(t)$. With the parameters of Fig. 3 and $\tau=0.1$, for which the oscillation amplitude and frequency are $A=0.428$ and $\omega=1.303$, the first Fourier amplitude (corresponding to frequency $\omega$ ) is $A_{1}=0.42$. Due to the cubic nonlinearity in the restoring force, in turn, the next significant amplitude corresponds to the third-harmonic component (frequency $3 \omega$ ), $A_{3}=0.0076$. Higher-harmonic amplitudes are even smaller. The first correction to harmonic motion, thus, is almost two orders of magnitude weaker than the main contribution, which reasonably justifies our analytical approximation. On the other hand, this modest contribution of higher-harmonic components to the overall motion markedly contrasts with the sizable phenomenology studied in section II., which is also a direct consequence of nonlinearity.

\section{Conclusions}

We have analyzed the dynamics of a mechanical oscillator whose periodic motion is sustained by a feedback force proportional to the oscillation velocity. The key ingredient that makes oscillations stable is a nonlinear dependence of damping with the oscillation amplitude, such that energy dissipation increases or decreases when the amplitude respectively grows or drops. This kind of nonlinearity, together with a cubic component in the restoring force, has been experimentally observed to occur in c-c beam micromechanical oscillators [6], which can therefore exhibit self-sustained motion under the action of linear velocity feedback.

Our emphasis was put on the effect of a time delay in the feedback force, assumed to originate in the electric circuit that reads, conditions, and reinjects the oscillation signal. The most significant consequence of this ingredient is that oscillation can be suppressed if the delay is large enough. It is well known that differential equations with time-delayed terms can exhibit either stationary oscillatory solutions or fixed rest points, depending on the delay [10]. Also, oscillation suppression (or "death") due to delays has been reported to occur in a variety of dynamical systems, such as synchronized limitcycle oscillators [11,12]. Here, this same occurrence has been characterized for a nonlinear mechanical system that can become relevant for the design of micromechanical time-keeping devices.

In connection with possible applications of this phenomenology, it is worth mentioning that classical-mechanical models such as Eq. (1) provide a suitable starting point for the description of micro- and nanomechanical machines. As length scales become smaller, however, the effects of thermal fluctuations and electrical noise cannot be further ignored $[13,14]$. From the viewpoint of our model, therefore, an important step forward would be to include noise in the theoretical analysis.

Acknowledgements - This project has been supported by Agencia Nacional de Promoción Científica y Tecnológica, Argentina, through grant PICT2014-1611. Fruitful collaboration with D. Antonio, S. Arroyo, Changyao Chen, D. Czaplewski, J. Guest, D. López, and F. Mangussi, as well as 
Papers in Physics, vol. 9, ART. 090003 (2017) / D. H. Zanette

discussions with S. Risau Gusmn, are gratefully acknowledged.

[1] B Yurke, D S Greywall, A N Pargellis, P A Busch, Theory of amplifier-noise evasion in an oscillator employing a nonlinear resonator, Phys. Rev. A 51, 4211 (1995).

[2] D Bishop, P Gammel, R Giles, The little machines that are making it big, Phys. Today $\mathbf{5 4}$, 38 (2001).

[3] K L Ekinci, M L Roukes, Nanoelectromechanical systems, Rev. Sci. Instrum. 76, 061101 (2005).

[4] D Antonio, D H Zanette, D López, Frequency stabilization in nonlinear micromechanical oscillators, Nat. Commun. 3, 806 (2012).

[5] D Antonio, D A Czaplewski, J R Guest, D López, S I Arroyo, D H Zanette, Nonlinearityinduced synchronization enhancement in micromechanical oscillators, Phys. Rev. Lett. 114, 034103 (2015).

[6] Changyao Chen, D H Zanette, J R Guest, D A Czaplewski, D López, Self-sustained micromechanical oscillator with linear feedback, Phys. Rev. Lett. 117, 017203 (2016).
[7] A H Nayfeh, D T Mook, Nonlinear Oscillations, Wiley, New York (2008).

[8] H B Peng, C W Chang, S Aloni, T D Yuzvinsky, A Zettl, Ultrahigh frequency nanotube resonators, Phys. Rev. Lett. 97, 087203 (2006).

[9] S I Arroyo, D H Zanette, Duffing revisited: Phase-shift control and internal resonance in self-sustained oscillators, Eur. Phys. J. B 89, 12 (2016).

[10] T Erneux, Applied Delay Differential Equations, Springer, New York (2009).

[11] D V Ramana Reddy, A Sen, G L Johnston, Time delay induced death in coupled limit cycle oscillators, Phys. Rev. Lett. 80, 5109 (1998).

[12] S H Strogatz, Death by delay, Nature 394 , 316 (1998)

[13] A N Cleland, M L Roukes, Noise processes in nanomechanical resonators, J. Appl. Phys. 92, 2758 (2002).

[14] P Ward, A Duwel, Oscillator phase noise: systematic construction of an analytical mode encompassing nonlinearity, IEEE Trans. Ultrason. Ferroelectr. Freq. Control 58, 195 (2011). 